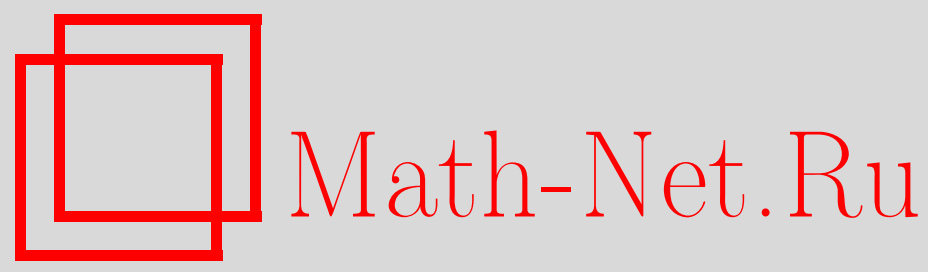

А. А. Ковалевский, О суммируемости решений нелинейных эллиптических уравнений с правыми частями из логарифмических классов, Матем. заметки, 2003, том 74, выпуск 5, 676-685

DOI: https://doi.org/10.4213/mzm300

Использование Общероссийского математического портала Math-Net.Ru подразумевает, что вы прочитали и согласны с пользовательским соглашением http://www . mathnet.ru/rus/agreement

Параметры загрузки:

IP: 52.205 .19 .152

26 апреля 2023 г., 18:03:48

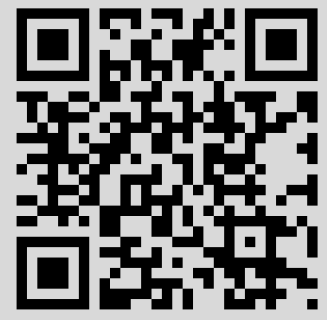




\section{О СУММИРУЕМОСТИ РЕШЕНИЙ НЕЛИНЕЙНЫХ ЭЛЛИПТИЧЕСКИХ УРАВНЕНИЙ С ПРАВЫМИ ЧАСТЯМИ ИЗ ЛОГАРИФМИЧЕСКИХ КЛАССОВ}

\section{А. А. Ковалевский}

Устанавливается существование принадлежащего некоторому соболевскому пространству слабого решения задачи Дирихле для нелинейного эллиптического уравнения второго порядка с правой частью из широкого класса функций, определяемого с помощью логарифмической функции.

Библиограф̆ия: 6 названий.

1. Введение и формулировка основного результата. Пусть $\Omega$ - ограниченное открытое множество в $\mathbb{R}^{n}(n \geqslant 2)$ и $p \in(1, n)$. Пусть $c_{1}, c_{2}$ - положительные постоянные, $g$ - неотрицательная функция из $L^{p /(p-1)}(\Omega)$, и пусть для любого $i \in\{1, \ldots, n\}$ $a_{i}-$ функция Каратеодори на $\Omega \times \mathbb{R}^{n}$. Будем предполагать, что для почти всех $x \in \Omega$ и любых $\xi \in \mathbb{R}^{n}$ имеют место неравенства

$$
\begin{aligned}
& \sum_{i=1}^{n}\left|a_{i}(x, \xi)\right| \leqslant c_{1}|\xi|^{p-1}+g(x), \\
& \sum_{i=1}^{n} a_{i}(x, \xi) \xi_{i} \geqslant c_{2}|\xi|^{p} .
\end{aligned}
$$

Кроме того, будем считать, что для почти всех $x \in \Omega$ и любых $\xi, \xi^{\prime} \in \mathbb{R}^{n}, \xi \neq \xi^{\prime}$, справедливо неравенство

$$
\sum_{i=1}^{n}\left[a_{i}(x, \xi)-a_{i}\left(x, \xi^{\prime}\right)\right]\left(\xi_{i}-\xi_{i}^{\prime}\right)>0 .
$$

Рассмотрим следующую задачу Дирихле:

$$
\begin{aligned}
& -\sum_{i=1}^{n} \frac{\partial}{\partial x_{i}} a_{i}(x, \nabla u)=f \quad \text { в } \Omega, \\
& u=0 \quad \text { на } \partial \Omega .
\end{aligned}
$$

Изучению разрешимости и свойств решений задачи (4) с $f$ из $L^{1}(\Omega)$ или из класса ограниченных мер посвящено достаточно большое число работ (см., например, [1]-[4]). Далее будем считать, что $f \in L^{1}(\Omega)$. 
ОПРЕДЕЛЕНИЕ 1. Слабым решением задачи (4) будем назьвать функцию $u \in$ $\stackrel{\circ}{W}^{1,1}(\Omega)$ такую, что вьполняются условия

1) для любого $i \in\{1, \ldots, n\} \quad a_{i}(x, \nabla u) \in L^{1}(\Omega)$;

2) для любой функции $\varphi \in C_{0}^{\infty}(\Omega)$

$$
\int_{\Omega}\left\{\sum_{i=1}^{n} a_{i}(x, \nabla u) D_{i} \varphi\right\} d x=\int_{\Omega} f \varphi d x
$$

По поводу этого определения см., например, [2].

Положим

$$
r=\frac{n(p-1)}{n-1} \text {. }
$$

В работе [2] показано, что если $p>2-1 / n$, то существует слабое решение задачи $(4)$, принадлежащее пространству $\stackrel{\circ}{W}^{1, \lambda}(\Omega)$ для любого $\lambda \in[1, r)$. При этом показатель $\lambda=r$, вообще говоря, недостижим. Однако, как установлено в [2], если $p>2-1 / n$ и $f \ln (1+|f|) \in L^{1}(\Omega)$, то слабое решение задачи (4), принадлежащее $\stackrel{\circ}{W^{1}, r}(\Omega)$, существует. Недавно в статье автора [5] было доказано сушествование слабого решения задачи (4), принадлежащего $\stackrel{\circ}{W}{ }^{1, r}(\Omega)$, при условиях $p \geqslant 2-1 / n$ и

$$
f[\ln (1+|f|)]^{\sigma} \in L^{1}(\Omega),
$$

где $\sigma \in((n-1) / n, 1)$. Оказьвается, что и этот результат допускает усиление. А именно, для того, чтобы утверждение о сушествовании слабого решения рассматриваемой задачи, принадлежащего $\stackrel{\circ}{W}^{1, r}(\Omega)$, оставалось в силе, достаточно в условии (5) множитель при $f$ заменить на произведение произвольного конечного числа последовательных суперпозиций логарифмической функции от $|f|$, взятьх в степени $(n-1) / n$, и следующей такой суперпозиции в степени $\sigma>(n-1) / n$. Доказательство данного факта является целью настоящей заметки.

Дадим точную формулировку основного результата статьи.

Определим последовательность чисел $s_{j}$ следуюшим образом:

$$
s_{1}=1, \quad s_{j}=e^{s_{j-1}}, \quad j=2,3, \ldots .
$$

Пусть теперь для любого $j \in \mathbb{N} b_{j}:\left[s_{j},+\infty\right) \rightarrow[0,+\infty)$ - функция такая, что

$$
b_{j}(s)=\underbrace{\ln \ldots \ln \ln }_{j} s, \quad s \in\left[s_{j},+\infty\right) .
$$

ТЕОРемА 1. Пусть $p \geqslant 2-1 / n$, и пусть выполняется условие: существуют $m \in \mathbb{N} u \sigma>(n-1) / n$ maкue, чmo

$$
f\left[\prod_{j=1}^{m} b_{j}\left(s_{j}+|f|\right)\right]^{(n-1) / n}\left[b_{m+1}\left(s_{m+1}+|f|\right)\right]^{\sigma} \in L^{1}(\Omega) .
$$

Тогда существует слабое решение задачи (4), принадлехащее $\stackrel{\circ}{W}^{1, r}(\Omega)$.

Доказательство теоремы 1 будет сведено к применению результатов работы [3] и устанавливаемого в следующем пункте нового результата о суммируемости функций, удовлетворяющих некоторому семейству интегральных неравенств (теорема 2). 
2. Суммируемость функций, подчиненных некоторым интегральным неравенствам. Прежде всего заметим, что если $j \in \mathbb{N}$ и $s>s_{j}$, то $b_{j}(s)>0$.

ЛЕмма 1. Пусть $и$-измеримая функиия на $\Omega$. Пусть $M>0, \tau>0, \varepsilon>1$, $m \in \mathbb{N}$. Пусть для любого $k>s_{m+1}$ справедливо неравенство

$$
\operatorname{meas}\{|u| \geqslant k\} \leqslant M k^{-\tau}\left[\prod_{j=1}^{m} b_{j}(k)\right]^{-1}\left[b_{m+1}(k)\right]^{-\varepsilon} \text {. }
$$

Тогда $и \in L^{\tau}(\Omega)$ и имеет место оценка

$$
\int_{\Omega}|u|^{\tau} d x \leqslant\left(e s_{m+2}\right)^{\tau} \text { meas } \Omega+\frac{2^{m+\varepsilon} e^{\tau} M}{\varepsilon-1} .
$$

ДокАЗАТЕЛЬСТво. Ограничимся предположением, что $m \geqslant 2$. Случай $m=1$ рассматривается аналогично.

Пусть $k_{0}$ - натуральное число такое, что $s_{m+1}<k_{0} \leqslant s_{m+1}+1$. Для любого $k \in \mathbb{N}$, $k \geqslant k_{0}$, положим

$$
\beta_{k}=\left[k \prod_{j=1}^{m-1} b_{j}(k)\right]^{-1}\left[b_{m}(k)\right]^{-\varepsilon} .
$$

Пусть $k \in \mathbb{N}, k \geqslant k_{0}$. В силу (7) имеем

$$
\operatorname{meas}\left\{|u| \geqslant e^{k}\right\} \leqslant M e^{-\tau k} \beta_{k} .
$$

Тогда

$$
\int_{\left\{e^{k} \leqslant|u|<e^{k+1}\right\}}|u|^{\tau} d x \leqslant M e^{\tau} \beta_{k} .
$$

Теперь, учитьвая, что в силу условия $\varepsilon>1$ имеем

$$
\sum_{k=k_{0}}^{\infty} \beta_{k} \leqslant \frac{2^{m+\varepsilon}}{\varepsilon-1}\left[b_{m}\left(k_{0}\right)\right]^{1-\varepsilon}
$$

из (9) выводим, что $u \in L^{\tau}(\Omega)$ и верна оценка

$$
\int_{\Omega}|u|^{\tau} d x \leqslant e^{\tau k_{0}} \operatorname{meas} \Omega+\frac{2^{m+\varepsilon} e^{\tau} M}{\varepsilon-1}\left[b_{m}\left(k_{0}\right)\right]^{1-\varepsilon} .
$$

Отсюда, принимая во внимание выбор числа $k_{0}$, получаем неравенство (8). Лемма доказана.

Положим $p^{*}=n p /(n-p)$. Известно (см., например, [6]), что существует положительная постоянная $c_{0}$, зависящая только от $n$ и $p$, такая, что для любой функции $u \in \stackrel{\circ}{W}^{1, p}(\Omega)$

$$
\left(\int_{\Omega}|u|^{p^{*}} d x\right)^{1 / p^{*}} \leqslant c_{0}\left(\int_{\Omega}|\nabla u|^{p} d x\right)^{1 / p} .
$$

Пусть для любого $k>0 \quad T_{k}-$ функция на $\mathbb{R}$ такая, что

$$
T_{k}(s)= \begin{cases}s, & \text { если }|s| \leqslant k, \\ k \operatorname{sign} s, & \text { если }|s|>k .\end{cases}
$$


ЛЕмма 2. Пусть $и$ - функиия на $\Omega$. Пусть $k>0 u T_{k}(u) \in \stackrel{\circ}{W^{1, p}}(\Omega)$. Тогда множество $\{|u| \geqslant k\}$ измеримо и справедливо неравенство

$$
\operatorname{meas}\{|u| \geqslant k\} \leqslant\left(c_{0} / k\right)^{p^{*}}\left(\int_{\Omega}\left|\nabla T_{k}(u)\right|^{p} d x\right)^{p^{*} / p} .
$$

ДокАЗАтЕЛЬСтво. Измеримость множества $\{|u| \geqslant k\}$ следует из равенства $\{|u| \geqslant k\}=\left\{\left|T_{k}(u)\right|=k\right\}$ и измеримости функции $T_{k}(u)$. В силу того же равенства имеем

$$
k^{p^{*}} \operatorname{meas}\{|u| \geqslant k\} \leqslant \int_{\Omega}\left|T_{k}(u)\right|^{p^{*}} d x .
$$

Отсюда и из неравенства (10), примененного к функции $T_{k}(u)$, выводим (11). Лемма доказана.

Лемма 3. Пусть $и$ - функция на $\Omega$, и пусть для любого $k>0 T_{k}(u) \in \stackrel{\circ}{W}^{1, p}(\Omega)$. Тогда для любих $N, k, k_{1}>0$ имеем

$$
\begin{gathered}
\operatorname{meas}\left\{\left|\nabla T_{N}(u)\right| \geqslant k\right\} \leqslant\left(c_{0} / k_{1}\right)^{p^{*}}\left(\int_{\Omega}\left|\nabla T_{k_{1}}(u)\right|^{p} d x\right)^{p^{*} / p} \\
+k^{-p} \int_{\Omega}\left|\nabla T_{k_{1}}(u)\right|^{p} d x
\end{gathered}
$$

ДокАЗАТЕЛЬСтво. Пусть $N, k, k_{1}>0$. Положим $E=\left\{|u|<k_{1},\left|\nabla T_{N}(u)\right| \geqslant k\right\}$. Ясно, что

$$
\operatorname{meas}\left\{\left|\nabla T_{N}(u)\right| \geqslant k\right\} \leqslant \operatorname{meas}\left\{|u| \geqslant k_{1}\right\}+\text { meas } E \text {. }
$$

Учитьвая, что $k \leqslant\left|\nabla T_{N}(u)\right|$ на $E$,

$$
\begin{array}{lll}
\nabla T_{N}(u)=\nabla T_{k_{1}}(u) & \text { п.в. на } & \{|u| \leqslant N\} \cap\left\{|u| \leqslant k_{1}\right\}, \\
\nabla T_{N}(u)=0 & \text { п.в. на } & \{|u|>N\},
\end{array}
$$

получаем

$$
k^{p} \text { meas } E \leqslant \int_{E}\left|\nabla T_{N}(u)\right|^{p} d x \leqslant \int_{\Omega}\left|\nabla T_{k_{1}}(u)\right|^{p} d x .
$$

Отсюда, из (13) и леммы 2 вьводим (12). Лемма доказана.

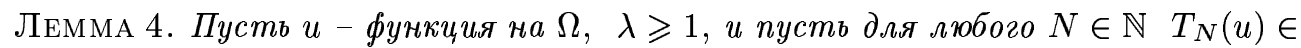
$W^{1, \lambda}(\Omega)$. Пусть последовательность $\left\{T_{N}(u)\right\}$ ограничена в $W^{1, \lambda}(\Omega)$. Тогда $u \in$ $W^{1, \lambda}(\Omega)$ и $T_{N}(u) \rightarrow$ и сильно в $W^{1, \lambda}(\Omega)$. 
ДокАЗАТЕЛЬСтво. В силу ограниченности последовательности $\left\{T_{N}(u)\right\}$ в $L^{\lambda}(\Omega)$, поточечной сходимости этой последовательности к $u$ в $\Omega$, леммы $Ф$ ату и теоремы Лебега о предельном переходе под знаком интеграла имеем $u \in L^{\lambda}(\Omega)$ и

$$
T_{N}(u) \rightarrow u \quad \text { сильно в } L^{\lambda}(\Omega) .
$$

Далее, пусть $i \in\{1, \ldots, n\}$. Используя тот факт, что для произвольных $k, k_{1} \in \mathbb{N}$, $k<k_{1}$,

$$
D_{i} T_{k}(u)=D_{i} T_{k_{1}}(u) \quad \text { п.в. на }\{|u| \leqslant k\},
$$

устанавливаем: существует функция $v_{i}: \Omega \rightarrow \mathbb{R}$ такая, что для любого $N \in \mathbb{N}$

$$
v_{i}=D_{i} T_{N}(u) \quad \text { п.в. на }\{|u| \leqslant N\} .
$$

Отсюда следует, что

$$
D_{i} T_{N}(u) \rightarrow v_{i} \quad \text { п.в. на } \Omega .
$$

Кроме того, из (15) и определения функций $T_{N}$ вытекает, что для любого $N \in \mathbb{N}$

$$
\left|D_{i} T_{N}(u)\right| \leqslant\left|v_{i}\right| \quad \text { п.в. на } \Omega .
$$

В силу ограниченности последовательности $\left\{D_{i} T_{N}(u)\right\}$ в $L^{\lambda}(\Omega),(16)$ и леммы Фату имеем $v_{i} \in L^{\lambda}(\Omega)$. Тогда, используя $(16),(17)$ и теорему Лебега о предельном переходе под знаком интеграла, получаем, что

$$
D_{i} T_{N}(u) \rightarrow v_{i} \quad \text { сильно в } L^{\lambda}(\Omega) .
$$

Из (14) и (18) следует, что существует обобщенная производная $D_{i} u, D_{i} u=v_{i}$ п.в. на $\Omega$. Теперь ясно, что $D_{i} u \in L^{\lambda}(\Omega)$ и $D_{i} T_{N}(u) \rightarrow D_{i} u$ сильно в $L^{\lambda}(\Omega)$. Поскольку это верно для любого $i \in\{1, \ldots, n\}$, то, учитывая (14), заключаем, что $u \in W^{1, \lambda}(\Omega)$ и $T_{N}(u) \rightarrow u$ сильно в $W^{1, \lambda}(\Omega)$. Лемма доказана.

ТЕОРема 2. Пусть $\Phi$ - неотрицательная измеримая функиия на $\Omega, m \in \mathbb{N}$, $\sigma>(n-1) / n$, u nусть

$$
\Phi\left[\prod_{j=1}^{m} b_{j}\left(s_{j}+\Phi\right)\right]^{(n-1) / n}\left[b_{m+1}\left(s_{m+1}+\Phi\right)\right]^{\sigma} \in L^{1}(\Omega) .
$$

Пусть $p \geqslant 2-1 / n, \quad$ и- функиия на $\Omega$, и пусть для любого $k>0 \quad T_{k}(u) \in \stackrel{\circ}{W^{1, p}}(\Omega)$ и справедливо неравенство

$$
\int_{\Omega}\left|\nabla T_{k}(u)\right|^{p} d x \leqslant \int_{\Omega} \Phi\left|T_{k}(u)\right| d x
$$

Tогда $u \in \stackrel{\circ}{W^{1, r}}(\Omega)$. 
ДокАЗАТЕЛЬСтво. Через $c_{i}, i=3,4, \ldots$, будем обозначать положительные постоянные, зависящие только от $n, p, m, \sigma$, meas $\Omega$ и нормы в $L^{1}(\Omega)$ функции

$$
\Phi\left[\prod_{j=1}^{m} b_{j}\left(s_{j}+\Phi\right)\right]^{(n-1) / n}\left[b_{m+1}\left(s_{m+1}+\Phi\right)\right]^{\sigma} .
$$

Определим числа $t_{j}$ следующим образом:

$$
t_{1}=4, \quad t_{j}=e^{t_{j-1}}, \quad j=2,3, \ldots, m+1 .
$$

Положим

$$
\gamma=\frac{p-1}{2 p}, \quad \gamma_{1}=\max \left(e^{1 / \gamma^{2}}, t_{m+1}\right) .
$$

Зафиксируем $k \geqslant \gamma_{1}$. Имеем $T_{k}(u) \in \stackrel{\circ}{W^{1, p}}(\Omega)$, и в силу (19) справедливо неравенство

$$
\int_{\Omega}\left|\nabla T_{k}(u)\right|^{p} d x \leqslant k^{\gamma} \int_{\Omega}\left|T_{k}(u)\right| d x+k \int_{\left\{\Phi>k^{\gamma}\right\}} \Phi d x .
$$

Используя (10) и неравенства Гёльдера и Юнга, получаем

$$
\begin{aligned}
k^{\gamma} \int_{\Omega}\left|T_{k}(u)\right| d x & \leqslant c_{0} k^{\gamma}(\text { meas } \Omega)^{\left(p^{*}-1\right) / p^{*}}\left(\int_{\Omega}\left|\nabla T_{k}(u)\right|^{p} d x\right)^{1 / p} \\
& \leqslant \frac{1}{p} \int_{\Omega}\left|\nabla T_{k}(u)\right|^{p} d x+\frac{p-1}{p} c_{3} k^{1 / 2} .
\end{aligned}
$$

Отсюда и из (20) следует, что

$$
\int_{\Omega}\left|\nabla T_{k}(u)\right|^{p} d x \leqslant c_{3} k^{1 / 2}+3 k \int_{\left\{\Phi>k^{\gamma}\right\}} \Phi d x .
$$

Оценим интеграл в правой части (21). Прежде всего заметим, что в силу неравенства $k \geqslant t_{m+1}$ и определения чисел $t_{j}$ для любого $j \in\{1, \ldots, m\}$ имеем

$$
b_{j}(k) \geqslant 4 \text {. }
$$

Очевидно, что имеет место следуюшее свойство:

$$
\text { если } s>k^{\gamma}, \text { то } b_{1}\left(s_{1}+s\right)>\gamma b_{1}(k) \text {. }
$$

Используя (22), (23) и неравенство $\ln k \geqslant 1 / \gamma^{2}$, устанавливаем, что если $s>k^{\gamma}$, то для любого $j \in\{2, \ldots, m+1\}$ справедливо неравенство $b_{j}\left(s_{j}+s\right)>b_{j}(k) / 2$. Этот факт и свойство (23) позволяют заключить, что

$$
\begin{aligned}
& {\left[\prod_{j=1}^{m} b_{j}\left(s_{j}+\Phi\right)\right]^{(n-1) / n}\left[b_{m+1}\left(s_{m+1}+\Phi\right)\right]^{\sigma}} \\
& \quad>\gamma^{m+\sigma}\left[\prod_{j=1}^{m} b_{j}(k)\right]^{(n-1) / n}\left[b_{m+1}(k)\right]^{\sigma} \text { на }\left\{\Phi>k^{\gamma}\right\} .
\end{aligned}
$$


Следовательно,

$$
\int_{\left\{\Phi>k^{\gamma}\right\}} \Phi d x \leqslant c_{4}\left[\prod_{j=1}^{m} b_{j}(k)\right]^{-(n-1) / n}\left[b_{m+1}(k)\right]^{-\sigma} .
$$

Заметим, что для произвольных $\lambda, s>0$ имеет место неравенство

$$
\lambda \ln s<s^{\lambda}
$$

Воспользовавшись этим, получаем

$$
\left[\prod_{j=1}^{m} b_{j}(k)\right]^{(n-1) / n}\left[b_{m+1}(k)\right]^{\sigma}<[2(m+\sigma)]^{m+\sigma} k^{1 / 2}
$$

Из неравенств $(21),(24)$ и (26) выводим, что для любого $k \geqslant \gamma_{1}$

$$
\int_{\Omega}\left|\nabla T_{k}(u)\right|^{p} d x \leqslant c_{5} k\left[\prod_{j=1}^{m} b_{j}(k)\right]^{-(n-1) / n}\left[b_{m+1}(k)\right]^{-\sigma}
$$

В силу леммы 2 и $(27)$ для любого $k>s_{m+1}$ имеем

$$
\operatorname{meas}\{|u| \geqslant k\} \leqslant c_{6} k^{-n(p-1) /(n-p)}\left[\prod_{j=1}^{m} b_{j}(k)\right]^{-(n-1) /(n-p)}\left[b_{m+1}(k)\right]^{-\sigma n /(n-p)}
$$

Отсюда, учитьвая неравенство $\sigma>(n-1) / n$ и используя лемму 1 , получаем, что $u \in$ $L^{n(p-1) /(n-p)}(\Omega)$.

Далее, положим

$$
\gamma_{2}=\gamma_{1}^{(n-1) /(n-p)}\left[\prod_{j=1}^{m} b_{j}\left(\gamma_{1}\right)\right]^{(n-1) / n(n-p)}\left[b_{m+1}\left(\gamma_{1}\right)\right]^{\sigma /(n-p)}
$$

и зафиксируем $k>\gamma_{2}$.

Пусть $\psi$ - функция на $\left(s_{m+1},+\infty\right)$ такая, что для любого $s \in\left(s_{m+1},+\infty\right)$

$$
\psi(s)=s^{n-1}\left[\prod_{j=1}^{m} b_{j}(s)\right]^{(n-1) / n}\left[b_{m+1}(s)\right]^{\sigma}
$$

Поскольку функция $\psi$ непрерывна, $\psi(s) \rightarrow+\infty$ при $s \rightarrow+\infty$ и $\psi\left(\gamma_{1}\right)<k^{n-p}$, то существует $k_{1}>\gamma_{1}$ такое, что

$$
\psi\left(k_{1}\right)=k^{n-p} .
$$


Отсюда следует, что

$$
\begin{aligned}
k_{1}^{-n(p-1) /(n-p)}\left[\prod_{j=1}^{m} b_{j}\left(k_{1}\right)\right]^{-(n-1) /(n-p)}\left[b_{m+1}\left(k_{1}\right)\right]^{-\sigma n /(n-p)} \\
=k^{-p} k_{1}\left[\prod_{j=1}^{m} b_{j}\left(k_{1}\right)\right]^{-(n-1) / n}\left[b_{m+1}\left(k_{1}\right)\right]^{-\sigma} \\
=k^{-r}\left[\prod_{j=1}^{m} b_{j}\left(k_{1}\right)\right]^{-1}\left[b_{m+1}\left(k_{1}\right)\right]^{-\sigma n /(n-1)}
\end{aligned}
$$

Кроме того, из (28) вытекает, что $k_{1}<k$ и $k^{n-p}<k_{1}^{n+m+\sigma}$. Используя последнее неравенство, устанавливаем, что для любого $j \in\{1, \ldots, m+1\}$

$$
b_{j}(k)<\frac{n+m+\sigma}{n-p} b_{j}\left(k_{1}\right) .
$$

Из леммы 3 и соотношений (27), (29), (30) выводим, что для любого $N \in \mathbb{N}$

$$
\operatorname{meas}\left\{\left|\nabla T_{N}(u)\right| \geqslant k\right\} \leqslant c_{7} k^{-r}\left[\prod_{j=1}^{m} b_{j}(k)\right]^{-1}\left[b_{m+1}(k)\right]^{-\sigma n /(n-1)} .
$$

Полученный результат, неравенство $\sigma>(n-1) / n$ и лемма 1 позволяют заключить, что для любого $N \in \mathbb{N}$

$$
\int_{\Omega}\left|\nabla T_{N}(u)\right|^{r} d x \leqslant c_{8}
$$

В силу условия $p \geqslant 2-1 / n$ имеем $r \geqslant 1$, а поскольку $p<n$, то $r<p$. Тогда для любого $N \in \mathbb{N}$ имеем $T_{N}(u) \in \stackrel{\circ}{W^{1}}, r(\Omega)$. Отсюда, из принадлежности функции $u$ пространству $L^{r}(\Omega),(31)$ и леммы 4 выводим, что $u \in \stackrel{\circ}{W}^{1, r}(\Omega)$. Тем самым теорема доказана.

3. Доказательство теоремы 1. Пусть условия теоремы 1 выполнены. В силу (1)-(3) и теоремы 6.1 из [3] существует функция $u$ на $\Omega$ такая, что для любого $k>0$ $T_{k}(u) \in \stackrel{\circ}{W^{1, p}}(\Omega)$ и для любых $\varphi \in \stackrel{\circ}{W^{1, p}}(\Omega) \cap L^{\infty}(\Omega), k>0$ и $k_{1}>k+\|\varphi\|_{L^{\infty}(\Omega)}$ справедливо неравенство

$$
\int_{\{|u-\varphi|<k\}}\left\{\sum_{i=1}^{n} a_{i}\left(x, \nabla T_{k_{1}}(u)\right)\left(D_{i} T_{k_{1}}(u)-D_{i} \varphi\right)\right\} d x \leqslant \int_{\Omega} f T_{k}(u-\varphi) d x .
$$

Отсюда, учитывая (2), получаем, что для любого $k>0$

$$
\int_{\Omega}\left|\nabla T_{k}(u)\right|^{p} d x \leqslant \frac{1}{c_{2}} \int_{\Omega}|f|\left|T_{k}(u)\right| d x .
$$

Теперь, учитывая (6) и используя теорему 2 , заключаем, что $u \in \stackrel{\circ}{W^{1, r}}(\Omega)$. Отсюда и из (1) вытекает, что для любого $i \in\{1, \ldots, n\} a_{i}(x, \nabla u) \in L^{1}(\Omega)$. Кроме того, в силу $(32)$ и следствия 4.3 из работы [3] для любой функции $\varphi \in C_{0}^{\infty}(\Omega)$ имеем

$$
\int_{\Omega}\left\{\sum_{i=1}^{n} a_{i}(x, \nabla u) D_{i} \varphi\right\} d x=\int_{\Omega} f \varphi d x .
$$

Таким образом, функция $u$ является слабым решением задачи (4). Теорема доказана. 
4. Заключительные замечания. Условие (6) на правую часть уравнения в рассматриваемой задаче (4) является лиш достаточньм для существования слабого решения этой задачи, принадлежашего $\stackrel{\circ}{W}{ }^{1, r}(\Omega)$. Действительно, покажем на примере оператора Лапласа (это соответствует случаю $p=2$ ), что слабое решение задачи (4), принадлежащее пространству $\stackrel{\circ}{W}^{1, r}(\Omega)$, может существовать и при более слабом ограничении на правую часть уравнения по сравнению с условием (6).

Пусть $\Omega=\left\{x \in \mathbb{R}^{n}:|x|<1\right\}$, и пусть $g_{1}$ - функция класса $C^{2}((0,+\infty))$ такая, что $g_{1}=0$ на $[1 / 2,+\infty)$ и для любого $s \in\left(0, e^{-e}\right)$

$$
g_{1}(s)=\left(\ln \frac{1}{s}\right)^{-(n-1) / n}\left(\ln \ln \frac{1}{s}\right)^{-(2 n-1) / n} .
$$

Пусть еще $u_{1}$ и $f_{1}-$ функции на $\Omega$ такие, что для любого $x \in \Omega \backslash\{0\}$

$$
u_{1}(x)=|x|^{2-n} g_{1}(|x|), \quad f_{1}(x)=(n-3)|x|^{1-n} g_{1}^{\prime}(|x|)-|x|^{2-n} g_{1}^{\prime \prime}(|x|) \text {. }
$$

Нетрудно проверить, что функция $u_{1}$ является слабьм решением задачи

$$
-\Delta u=f_{1} \quad \text { в } \Omega, \quad u=0 \text { на } \partial \Omega,
$$

при этом $u_{1} \in \stackrel{\circ}{W^{1}}, r(\Omega) ;$ для любого $\sigma \in(0,(n-1) / n)$

$$
f_{1}\left[\ln \left(1+\left|f_{1}\right|\right)\right]^{(n-1) / n}\left[\ln \ln \left(e+\left|f_{1}\right|\right)\right]^{\sigma} \in L^{1}(\Omega),
$$

HO

$$
f_{1}\left[\ln \left(1+\left|f_{1}\right|\right)\right]^{(n-1) / n}\left[\ln \ln \left(e+\left|f_{1}\right|\right)\right]^{(n-1) / n} \notin L^{1}(\Omega)
$$

и, следовательно, функция $f_{1}$ не удовлетворяет условию (6).

В общей ситуации, рассмотренной в этой статье, потеря точности в условии на правую часть уравнения, обеспечивающем существование слабого решения задачи (4) из $\stackrel{\circ}{W}^{1, r}(\Omega)$, происходит из-за того, что в основной теореме 2 при оценке интегралов функции $\Phi$ по множествам $\left\{\Phi>k^{\gamma}\right\}$ (см. (24)) интегралы функции

$$
\Phi\left[\prod_{j=1}^{m} b_{j}\left(s_{j}+\Phi\right)\right]^{(n-1) / n}\left[b_{m+1}\left(s_{m+1}+\Phi\right)\right]^{\sigma}
$$

по тем же множествам оцениваются сверху единой константой, тогда как последовательность этих интегралов стремится к нулю при $k \rightarrow \infty$. Однако для функции $\Phi$ общего положения по-видимому невозможно дать квалифицированную оценку точности этого стремления к нулю в зависимости от $k$ и тем самым воспользоваться надлежащим образом дополнительным множителем, сходящимся к нулю при $k \rightarrow \infty$, в правой части неравенства, огрублением которого явилось неравенство (24). Это оправдывает оценку упомянутых интегралов функции (33) только константой, хотя последнее, как уже было сказано, и приводит к некоторой потере точности в условии, обеспечивающем существование слабого решения рассматриваемой задачи, принадлежащего пространству $\stackrel{\circ}{W}{ }^{1, r}(\Omega)$. 
Наконец, приведем пример, когда правая часть уравнения имеет определенную "логарифмическую” суммируемость, но слабое решение соответствующей задачи Дирихле не принадлежит $\stackrel{\circ}{W}^{1, r}(\Omega)$.

Пусть $\Omega=\left\{x \in \mathbb{R}^{n}:|x|<1\right\}$, и пусть $g_{2}$ - функция класса $C^{2}((0,+\infty))$ такая, что $g_{2}=0$ на $[1 / 2,+\infty)$ и для любого $s \in\left(0, e^{-1}\right)$

$$
g_{2}(s)=\left(\ln \frac{1}{s}\right)^{-(n-1) / n} .
$$

Пусть еще $u_{2}$ и $f_{2}-$ функции на $\Omega$ такие, что для любого $x \in \Omega \backslash\{0\}$

$$
u_{2}(x)=|x|^{2-n} g_{2}(|x|), \quad f_{2}(x)=(n-3)|x|^{1-n} g_{2}^{\prime}(|x|)-|x|^{2-n} g_{2}^{\prime \prime}(|x|) \text {. }
$$

Тогда функция $u_{2}$ является слабым решением задачи

$$
-\Delta u=f_{2} \quad \text { в } \Omega, \quad u=0 \text { на } \partial \Omega \text {. }
$$

При этом для любого $\lambda \in[1, r)$ имеем $u_{2} \in \stackrel{\circ}{W^{1, \lambda}}(\Omega)$, но $\left|\nabla u_{2}\right| \notin L^{r}(\Omega)$ и, следовательно, $u_{2} \notin \stackrel{\circ}{W} 1, r(\Omega)$. Кроме того, для любого $\sigma \in(0,(n-1) / n)$ имеем $f_{2}\left[\ln \left(1+\left|f_{2}\right|\right)\right]^{\sigma} \in L^{1}(\Omega)$, но $f_{2}\left[\ln \left(1+\left|f_{2}\right|\right)\right]^{(n-1) / n} \notin L^{1}(\Omega)$.

\section{СПИСОК ЦИТИРОВАННОЙ ЛИТЕРАТУРЫ}

[1] Boccardo L., Gallouët T. Non-linear elliptic and parabolic equations involving measure data // J. Funct. Anal. 1989. V. 87. P. 149-169.

[2] Boccardo L., Gallouët T. Nonlinear elliptic equations with right hand side measures // Comm. Partial Differential Equations. 1992. V. 17. P. 641-655.

[3] Bénilan Ph., Boccardo L., Gallouët T., Gariepy R., Pierre M., Vazquez J. L. An $L^{1}$-theory of existence and uniqueness of solutions of nonlinear elliptic equations // Ann. Scuola Norm. Sup. Pisa Cl. Sci. 1995. V. 22. P. 241-273.

[4] Boccardo L., Gallouët T. Summability of the solutions of nonlinear elliptic equations with right hand side measures // J. Convex Analysis. 1996. V. 3. P. 361-365.

[5] Ковалевский А. А. О суммируемости решений нелинейных эллиптических уравнений с правыми частями из классов, близких к $L^{1}$ // Матем. заметки. 2001. Т. 70. № 3. С. 375-385.

[6] Gilbarg D., Trudinger N. S. Elliptic partial differential equations of second order. Berlin: Springer-Verlag, 1983.

Институт прикладной математики и механики НАН Украины

Поступило

E-mail: alexkvl@iamm.ac.donetsk.ua

22.06 .2001

Исправленный вариант

08.04 .2002 